\title{
THE USE OF PERKINS' TRACTION IN THE TREATMENT OF FEMORAL SHAFT FRACTURES
}

\author{
RICHARD A. BUXTON \\ From Princess Margaret Hospital, Swindon
}

\begin{abstract}
The treatment of fractures of the femoral shaft by traction may delay union and produce stiffness of the knee. The technique of Perkins' method is described and the results reported in $\mathbf{5 0}$ cases. All patients had at least 120 degrees of flexion of the knee when traction was discontinued, and 47 patients were considered to have clinical and radiological union of the fracture by 12 weeks. The main complication of the method was loosening of the Denham pin or infection of the pin-track in the proximal tibia.

It is suggested that Perkins' traction is an excellent form of treatment for fractures of the femoral shaft, and has several advantages over other forms of traction.
\end{abstract}

The exponents of internal fixation for the treatment of femoral shaft fracture quote delayed union and stiffness of the knee as complications of conservative methods (Müller et al. 1979). Indeed, a review of the literature reveals a high incidence of these complications when Russell's or balanced skeletal traction is used. Winant (1949), in a series of 68 patients treated in balanced suspension, found that stiffness of the knee was common, and the average time taken for union was over six months. Anderson (1967), in a series of 56 adults followed up for one to five years, reported that only 34 patients ( 65 per cent) had knee flexion of more than 120 degrees and six patients required operative intervention for non-union, three due to interposition of muscle and three due to over-distraction at the fracture site. Nichols (1963), in a comparison of different methods of treatment in 181 cases, found that only 41 per cent of patients treated by traction regained a range of flexion from 0 to 90 degrees, and that 30 per cent took longer than six months for the fracture to unite.

George Perkins first described, in his Robert Jones lecture, his method of treatment for femoral shaft fractures using the "split-bed" (Perkins 1953), and later published an account of the method in his book Fractures and dislocations (1958). He claimed that active movement of the muscles caused compression at the fracture site, promoted union and eliminated stiffness at the knee. He agreed that external splintage was harmful, frequently causing stiffness and that the bone ends could be distracted resulting in fibrous union (Perkins 1970). Perkins never published his results. His method of treatment is not widely used, but it has been used in Princess Margaret Hospital, Swindon, for several years. This paper reports a study of 50 patients with fractures of the femoral. shaft treated by Perkins' traction between 1969 and 1977.

\section{TECHNIQUE OF PERKINS METHOD}

The beds used have a mattress and base divided in a ratio of $3: 2$, the larger section being at the head of the bed.

With the patient under general anaesthesia, a Denham pin is passed through the upper tibia, and traction is applied through two cords which pass over pulleys at the foot of the bed to weights. A total weight of about 20 pounds ( 10 kilograms) is necessary for an adult male, and the foot of the bed is elevated one inch for each pound of traction applied. The length of the limb is checked with a tape-measure and weights added or removed as necessary. Rotation is controlled by elevating or depressing one or other of the pulley attachments. A pillow is placed under the thigh to maintain the natural anterior bowing of the femur at the fracture site.

Active physiotherapy should be started as soon as possible after admission, unless the patient has other injuries which preclude this. On the first day, some light analgesia may be given. A further pillow should be placed under the thigh and the patient positioned with the knee over the "split" in the mattress. While one physiotherapist supports the injured limb, a second removes the mattress and board from under the patient's leg. The mattress may be used as an additional back rest (Fig. 1). The patient is encouraged to flex the knee as far as possible against resistance under the heel, and then to extend the knee. This is continued for half an hour on the first day. It is important to perform flexion against resistance otherwise the movement is painful: a canvas sling for the heel, suspended on a spring from a Balkan beam may be used. As the patient gains confidence and control of his muscles, he needs less manual support from the physiotherapist. After a week he is usually able to flex and extend the knee unassisted. The time spent exercising should increase progressively each day up to a maximum of seven or eight hours at two weeks. While the knee is actively flexed and extended, movement can also be seen and felt at the fracture site, until about the fourth week when consolidation of callus occurs. Surprisingly, the patient experiences little or no discomfort. At night, the mattress is replaced, to support the limb. 
In patients with an ipsilateral fracture of the tibia, a below-knee cast incorporating the Denham pin may be used and management of the femoral fracture proceeds in the usual way.

A radiograph should be taken after traction is set up. Frequent

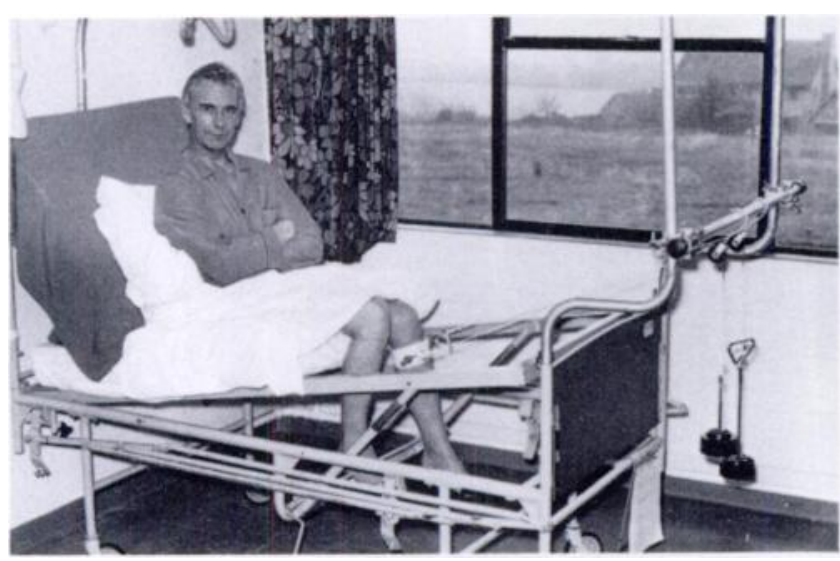

Fig. 1

Patient at four weeks, flexing the knee in a split-bed.

follow-up films are unnecessary, and radiographs need only be repeated at six weeks and 12 weeks from the time of fracture.

\section{MATERIAL AND METHODS}

The notes and radiographs of all patients with femoral shaft fractures treated by Perkins' method at Princess Margaret Hospital, Swindon, between 1969 and 1977 were examined to determine the following: cause of injury, site and type of fracture, presence of other injuries, the time when active physiotherapy was started, total length of time in traction, presence of any angulation on the final radiograph and any complications which occurred.

Patients were requested to attend for follow-up examination and the first 50 to attend were included in the survey. Detailed clinical examination was performed to determine presence or absence of a limp, the shape, alignment and length of the leg; the range of movement of the hip, the knee and the ankle was measured using a goniometer. The knee was also examined for ligamentous laxity, the presence of any extension "lag" and any problems related to the pin-track.

There were 40 male patients and 10 female. The age range was from 11 to 70 years, 27 being in their teens

Table I. Site and type of the 54 fractures of the femoral shaft

\begin{tabular}{|lr|lr|}
\hline \multicolumn{1}{|c|}{ Site } & \multicolumn{2}{|c|}{ Type } \\
\hline Uppermost third & 4 & & \\
Uppermost and middle thirds & 11 & Transverse & 26 \\
Middle third & 27 & Short oblique & 10 \\
Middle and lowermost thirds & 9 & Spiral & 3 \\
Lowermost third & 3 & Comminuted & 15 \\
\hline
\end{tabular}

and 12 in their twenties. Forty-five patients were examined by the author and five by consultants at Princess Margaret Hospital.

\section{RESULTS}

\section{Analysis of notes and radiographs}

In 33 patients the fracture had occurred in the right femur, and in 13 in the left; four patients had had bilateral fractures (Table I). Of the total of 54 fractures, eight had been compound and 46 closed. The majority ( 72 per cent) had been caused by road traffic accidents.

Thirty-one patients had had other injuries, 13 having had a significant injury to the same limb. These included four plateau fractures of the tibia, one fracture of the uppermost third of the tibia, entering the joint, two compound fractures of the patella, one compound injury into the knee, two cases of fracture of the calcaneum, one subtalar dislocation, one ankle dislocation, one transmetatarsal dislocation and one fracture of the medial malleolus.

Start of physiotherapy. Active flexion of the knee had been started in 37 patients within one week, and in 11 patients within three weeks. Two patients had had severe head and chest injuries, and active physiotherapy had been delayed for four and nine weeks.

Time in traction. Forty-seven patients ( 94 per cent) had been judged to have clinical and radiological union of the fracture by 12 weeks and had been mobilised on crutches, either non-weight-bearing, partial weightbearing or in a few cases full weight-bearing. In three patients with high-velocity injuries union had been delayed till 16, 18 and 28 weeks.

Complications. Five patients had developed the clinical syndrome of fat embolism: three were patients with multiple fractures and two had had bilateral femoral shaft fractures; all recovered. One patient had had a deep vein thrombosis, confirmed by venography, and

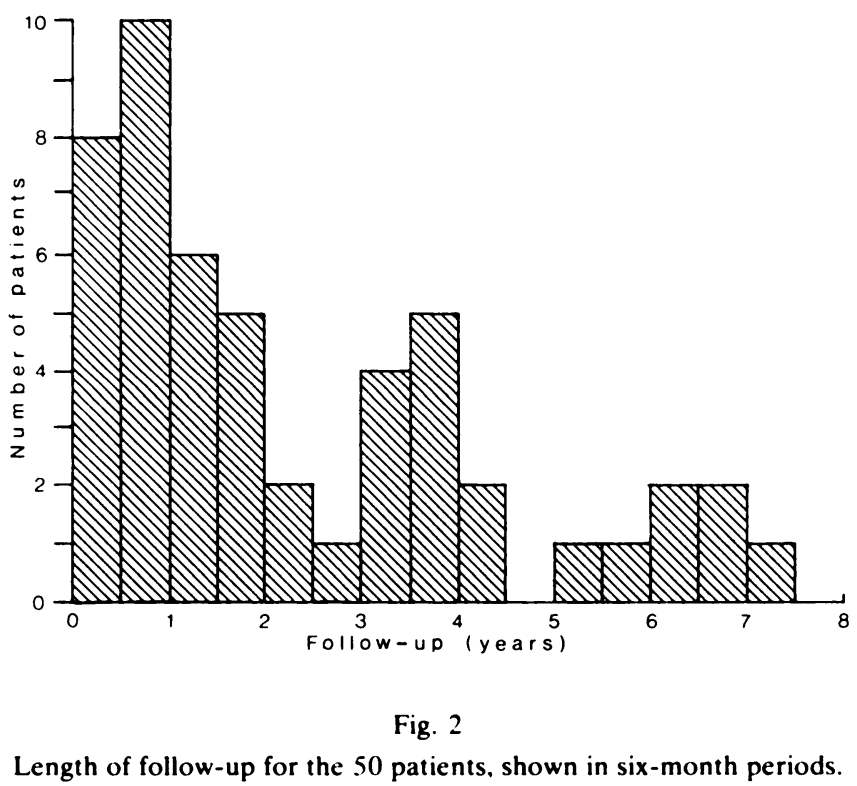




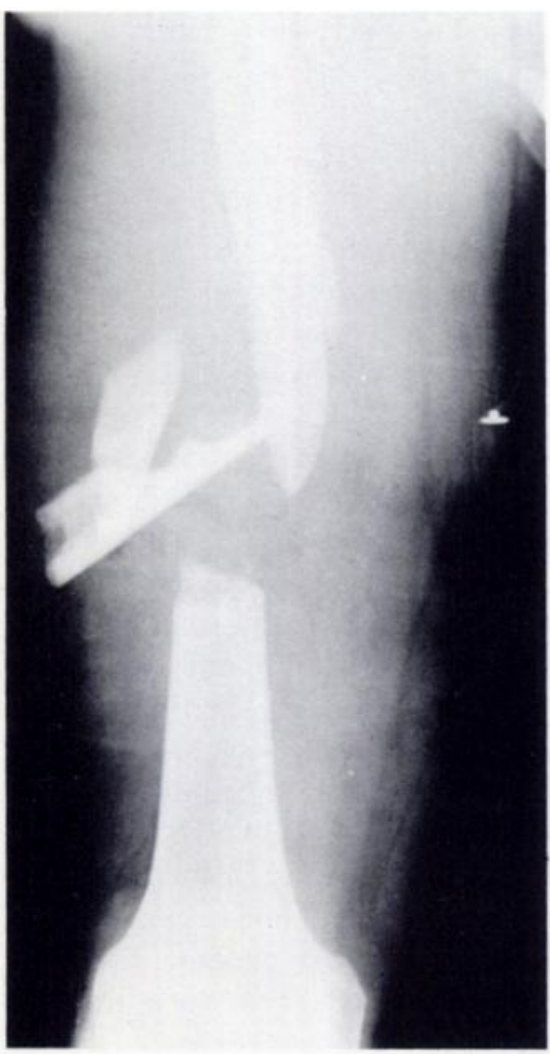

Fig. 3

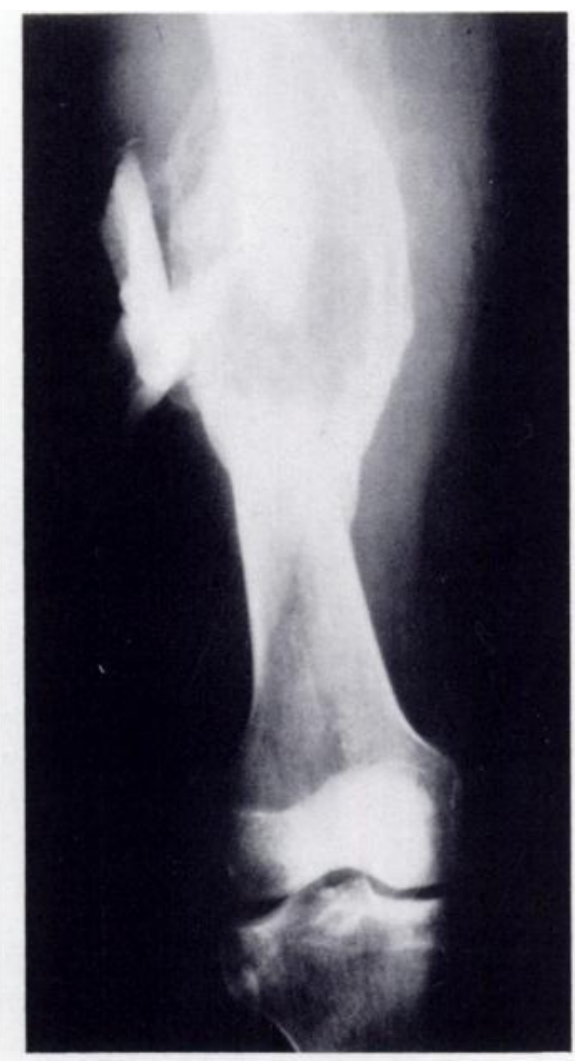

Fig. 4

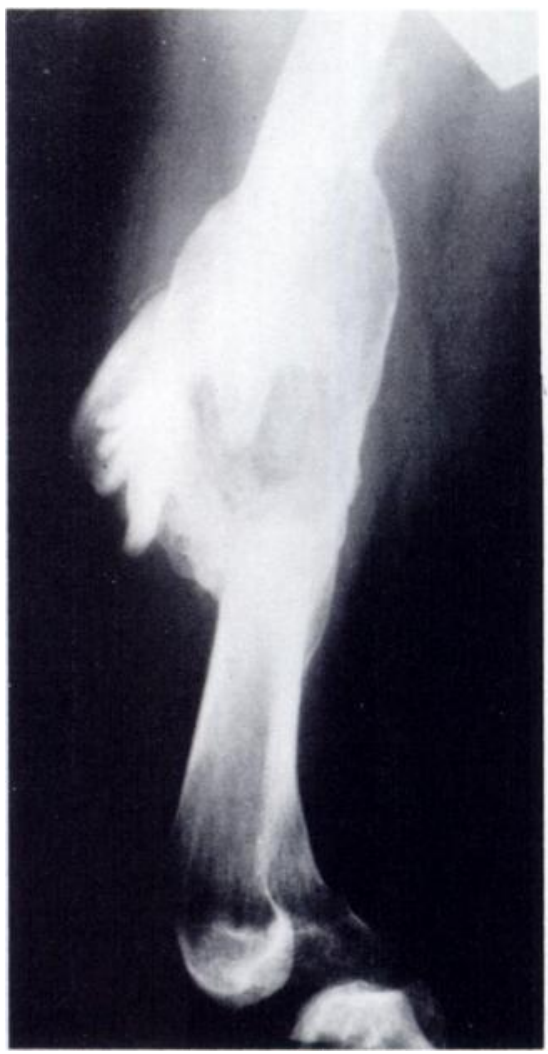

Fig. 5

Figure 3-A compound comminuted fracture of the femur sustained by a 24-year-old man in a road traffic accident. Active flexion of the knee was started one week after injury. Figures 4 and 5 -The same fracture, showing abundant callus, 12 weeks after injury when partial weight-bearing on crutches was started.

one other patient a pulmonary embolus without clinical evidence of deep vein thrombosis: both had been treated with anticoagulants and recovered. Four patients had suffered refracture within two weeks of mobilisation, caused by a fall or by excessive load-bearing on the affected leg; they all had healed rapidly with a further period of traction or mobilisation in a spica cast. Eighteen patients ( 36 per cent) had had a problem related to the pin-track: eight with proven staphylococcal infections, six with inflammation and four with a loose pin. The infections, including one case with a sequestrum, had all resolved with antibiotics on removal of the pin. When the pin worked loose, it was either removed early or replaced farther down the tibial shaft.

\section{Follow-up assessment}

Follow-up ranged from three months to seven and a quarter years from the time of fracture, with a mean of two years five months (Fig. 2).

Leg length. Thirty-three patients (66 per cent) had no discrepancy in leg length, while 14 (28 per cent) had a discrepancy of less than two centimetres; two patients had a shortening of three centimetres and one patient, with a transverse fracture at the junction of the uppermost and middle thirds of the shaft, had shortening of four centimetres.
Knee flexion. Thirty patients (60 per cent) had a full range of movement when compared with the opposite knee. No patient had flexion less than 120 degrees. Flexion continued to improve up to six months from cessation of traction. Of the patients examined after this time and who had less than full flexion, all but one had suffered comminuted fractures of the femoral shaft (Figs 3 to 7). Only one patient, with a comminuted fracture of the middle and lowermost thirds of the shaft, had an extension lag of 10 degrees. Five patients had minimal laxity of the collateral ligament, but none complained of instability of the knee.

Angulation at the fracture site. The radiographs of all patients were examined, except in four cases where the films had been destroyed. The angulation at the site of the healed fracture was measured in both the anteroposterior and lateral views and the maximum recorded. Thirty patients had no angulation. Two patients, both with fractures of the uppermost third, had a varus angulation of 15 degrees and 20 degrees. The remaining eighteen had an anterior angulation. The normal anterior bow of the femur measures 10 to 20 degrees and this should be taken into account when interpreting the results. In none of the patients was the angulation detectable clinically. 


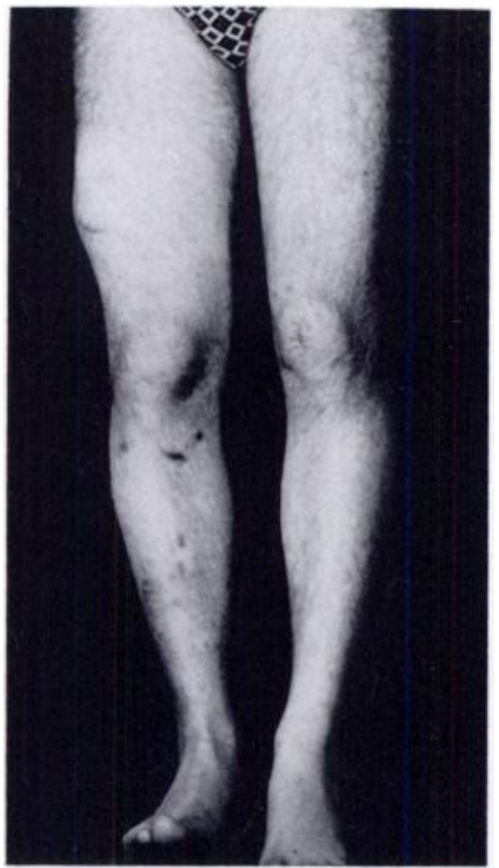

Fig. 6

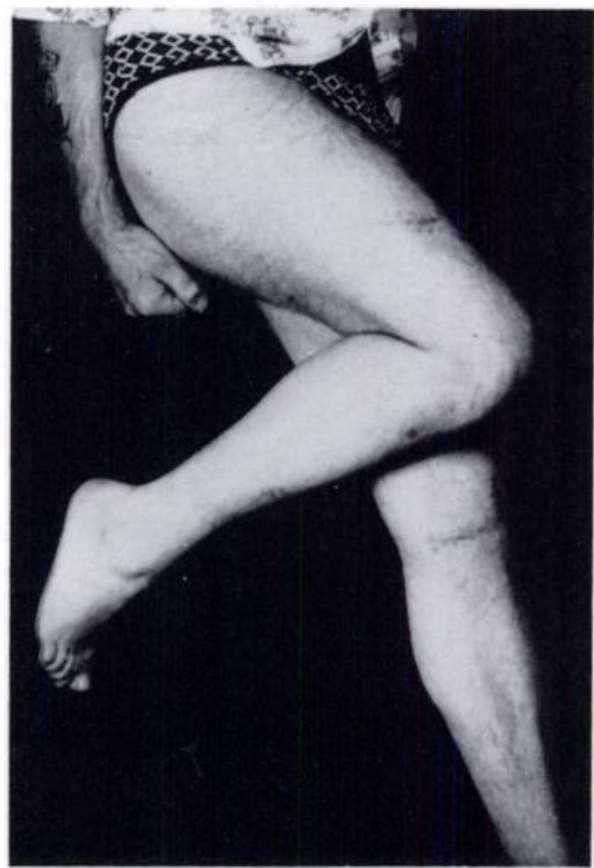

Fig. 7

Figures 6 and 7 - The same patient showing two centimetres of shortening and 120 degrees of flexion.

Four patients had slight lateral rotation deformity detectable clinically, but in none was it more than 10 degrees.

\section{DISCUSSION}

It is a sound principle of treatment that movement and physiological compression enhance union of a fracture (Charnley 1961; Sarmiento 1970; McKibbin 1978) and yet "conservative treatment" of fractures of the femoral shaft often means virtual immobilisation of the leg in cumbersome splints and traction devices. This report has shown that Perkins' method, employing early active flexion of the knee without external splintage, ensures an excellent range of movement, promotes union by early abundant callus formation, and has fewer serious complications than other methods of traction (Winant 1949; Nichols 1963; Anderson 1967).

Perkins' traction is easy to set up and maintain. Leg length is estimated by clinical measurement and adjusted by altering the weights. Three patients had unacceptable shortening of three and four centimetres, and more weight should have been applied in these cases. Rotation is controlled by the position of the traction cords, and is effectively abolished once the patient flexes the knee to 90 degrees. Skin sores are non-existent because no splint is used and the patients are very mobile in bed. Although significant varus did not occur in this series, it is a likely complication in fractures of the uppermost third and may be controlled by abduction of the leg with sling countertraction medially if required. Radiographic evidence of anterior angulation was found to be of no cosmetic or functional significance, in spite of the fact that some angles were very large. Apley (1977) has stressed the importance of not just treating the radiographic appearance of these fractures, it is the function of the limb as a whole which is important.

Early movement of the knee prevents the development of patellofemoral and capsular adhesions which occur with immobilisation of an injured limb; adhesions between callus and the quadriceps are also minimised. By starting full active movement of the muscles over the fracture very soon after injury abundant early bridging callus is formed. In three patients with high-velocity injuries, union was delayed for over 12 weeks, but it is doubtful whether any other form of treatment could have promoted earlier union.

The main complication was infection of the pin-track or loosening of the pin, which is subject to a lot of movement. Infection is minimised by keeping the pin-track site well covered with sterile dressings and a firm crêpe bandage. If a pin works loose, it may be resited farther down the tibial shaft.

Although all the patients in this series were treated in traction for 12 weeks, it may be possible to change to a cast-brace after four weeks, by which time most patients have abundant callus, firm union, and 90 degrees of flexion at the knee. 


\section{REFERENCES}

Anderson RL. Conservative treatment of fractures of the femur. J Bone Joint Surg [Am] 1967:49-A:1371-5.

Apley AG. System of orthopaedics and fractures. 5th ed. London: Butterworths, 1977.

Charnley J. The closed treatment of common fractures. 3rd ed. Edinburgh: E \& S Livingstone Ltd. 1961.

McKibbin B. The biology of fracture healing in long bones. J Bone Joint Surg [Br] 1978;60-B:150-62.

Müller ME, Allgöwer M, Schneider R, Willineger H. Manual of internal fixation. 2nd ed. Berlin: Springer-Verlag. 1979.

Nichols PJR. Rehabilitation after fractures of the shaft of the femur. J Bone Joint Surg [Br] 1963;45-B:96-102.

Perkins G. Rest and movement. J Bone Joint Surg [Br] 1953;35-B:521-39.

Perkins G. Fractures and dislocations. London: Athlone Press, 1958.

Perkins G. The ruminations of an orthopaedic surgeon. London: Butterworth and Company, 1970.

Sarmiento A. A functional below-the-knee brace for tibial fractures. J Bone Joint Surg [Am] 1970;52-A:295-311.

Winant EM. The use of skeletal traction in the treatment of fractures of the femur. J Bone Joint Surg [Am] 1949;31-A:87-93. 